\title{
Simvastatin intervention mitigates hypercholesterolemia-induced alveolar bone resorption in rats
}

\author{
XIAOLI GAO ${ }^{1,2}$, JIANHUA ZHOU ${ }^{3}$, YUANYUAN BIAN ${ }^{1,2}$, SHENGYUN HUANG $^{2}$ and DONGSHENG ZHANG ${ }^{1,2}$ \\ ${ }^{1}$ Department of Oral and Maxillofacial Surgery, School and Hospital of Stomatology, Shangdong University and \\ Shandong Provincial Key Laboratory of Oral Tissue Regeneration and Shandong Engineering Laboratory for Dental \\ Materials and Oral Tissue Regeneration, Jinan, Shandong 250012; ${ }^{2}$ Department of Oral and Maxillofacial Surgery, \\ Shandong Provincial Hospital Affiliated to Shandong First Medical University, Jinan, Shandong 250021; \\ ${ }^{3}$ Department of Stomatology, Qingdao Municipal Hospital, Qingdao, Shandong 266071, P.R. China
}

Received July 28, 2020; Accepted March 18, 2021

DOI: $10.3892 /$ etm.2021.10060

\begin{abstract}
Simvastatin promotes bone formation and increases bone mineral density in patients with hyperlipidemia and ameliorates hypercholesterolemia-induced microstructure changes in the jaw bone of animals. However, whether and how treatment with simvastatin can modulate the hypercholesterolemia-induced alveolar bone resorption is unclear. The present study aimed to examine the therapeutic efficacy and potential mechanisms of simvastatin application in hypercholesterolemia-induced alveolar bone resorption. The association between hyperlipidemia and alveolar bone resorption in 100 patients with periodontitis was examined. Additionally, male Sprague-Dawley rats were fed a standard rodent chow (NC) for 32 weeks or a high cholesterol diet (HCD) for 24 weeks. The HCD-fed rats were randomized, continually fed with HCD and treated with vehicle saline (HC) or simvastatin by gavage ( $5 \mathrm{mg} / \mathrm{kg}$; SIM, n=10/group) for 8 weeks. The morphological changes to alveolar bone resorption in rats were analyzed by linear measurements. The relative levels of osteoprotegerin $(\mathrm{OPG})$, receptor activator of nuclear factor- $\mathrm{\kappa B}$ ligand RANKL, nuclear factor- $\kappa \mathrm{B}$ (NF- $\kappa \mathrm{B})$, microtubule-associated protein 1 light chain 3 (LC3) and p62 in the alveolar bone tissues were determined by reverse transcription-quantitative PCR and/or immunohistochemistry. Sulcus bleeding index (SBI), clinical attachment loss (CAL), probing depth (PD) and the distance of cemantoenamel junction-alveolar bone crest (CEJ-ABC) in patients with hyperlipidemia were significantly
\end{abstract}

Correspondence to: Dr Dongsheng Zhang, Department of Oral and Maxillofacial Surgery, School and Hospital of Stomatology, Shangdong University and Shandong Provincial Key Laboratory of Oral Tissue Regeneration and Shandong Engineering Laboratory for Dental Materials and Oral Tissue Regeneration, 44-1 Wenhua Road West, Jinan, Shandong 250012, P.R. China

E-mail: ds63zhang@sdu.edu.cn

Key words: hypercholesterolemia, simvastatin, alveolar bone, autophagy greater than that in the controls $(\mathrm{P}<0.001)$. The levels of hyperlipidemia were positively correlated with the values of SBI, CAL, PD and CEJ-ABC in this population. Compared with the $\mathrm{NC}$ rats, higher levels of alveolar bone resorption, $\mathrm{NF}-\mathrm{\kappa B}$ expression, higher ratios of RANKL/OPG mRNA transcripts and LC3 to p62 expression were detected in the alveolar bone tissues of HC group. Simvastatin intervention significantly mitigated hypercholesterolemia-induced alveolar bone loss and RANKL mRNA transcription, but increased the ratios of LC3/p62 protein expression in the alveolar bone tissues of rats. Hyperlipidemia is associated with alveolar bone resorption and simvastatin treatment alleviated the hypercholesterolemia-related alveolar bone loss by down-regulating the NF- $\mathrm{kB}$ expression.

\section{Introduction}

Periodontitis is the most common dental disease globally and one of the major threats for tooth loss worldwide (1). Periodontitis can damage the supporting tissues of the teeth, which can promote alveolar bone resorption, and periodontitis can be worsened by the alveolar bone loss (2). Periodontitis progression has been attributed to hyperlipidemia and particularly to hypercholesterolemia (3). Hyperlipidemia is associated with the development of severe periodontitis and high degrees of alveolar bone loss in animal models (4). However, little information is available about whether hyperlipidemia is related to the severity of alveolar bone resorption in Chinese patients with periodontitis. Additionally, it is unclear how hyperlipidemia promotes periodontitis-related alveolar bone resorption. Understanding the mechanisms by which hyperlipidemia promotes alveolar bone resorption will be of significance in uncovering new therapeutic targets for the development of novel periodontitis treatments.

Hyperlipidemia can cause chronic inflammation, as lipids can engage toll-like receptors (TLRs)-2 and 4 to activate the nuclear factor- $\mathrm{\kappa B}(\mathrm{NF}-\mathrm{\kappa B})$ signaling to produce pro-inflammatory cytokines, including tumor necrosis factor (TNF)- $\alpha$, interleukin (IL)-1 $\beta$, IL-6 and receptor activator of NF- $\mathrm{KB}$ ligand (RANKL). These pro-inflammatory cytokines can enhance osteoclastogenesis and lead to bone resorption, 
which is suppressed by osteoprotegerin (OPG) produced by osteoblasts. Osteoclastogenesis is also regulated by autophagic components, including beclin1 and p62, as well as inflammatory signaling $(5,6)$. Given that autophagy is crucial for the survival and function of many types of cells, including osteoclasts (7), modulation of hypercholesterolemia, inflammation and osteoclastogenesis, as well as osteoclast autophagy, may be valuable for the management of hyperlipidemia-induced alveolar bone resorption and periodontitis.

Statins can inhibit 3-hydroxy-3-methylglutaryl coenzyme A (HMG-CoA) reductase to lower serum lipid levels and have been demonstrated to benefit patients with hyperlipidemia in the clinic (8). Previous studies have shown that statins not only reduce serum lipid levels, but also reduce inflammation and benefit bone metabolism (9). A recent study indicated that treatment with statins promoted bone formation and increased bone mineral density (BMD) in patients with hyperlipidemia and ameliorated hypercholesterolemia-related microstructure changes in the jaw bone of animals (10). However, whether and how treatment with simvastatin could modulate hypercholesterolemia-related alveolar bone resorption has not been clarified.

The present study aimed to investigate how hyperlipidemia was related to the severity of alveolar bone resorption in Chinese patients with periodontitis and examined the therapeutic efficacy and potential mechanisms of simvastatin intervention for alveolar bone resorption in hypercholesterolemic rats.

\section{Materials and methods}

Subjects. The present study was conducted in accordance with the guidelines in the Helsinki Declaration (11) and the experimental protocol was approved by the Ethics Committee of Shandong Provincial Hospital (approval no. SWYX: No. 2021-013). This study enrolled 100 patients (age range, 28 to 65 years) with periodontitis at the Shandong Provincial Hospital between March and June 2018. Individual patients with periodontitis were diagnosed on the basis of periodontal examinations, which included assessment of plaque, tartar buildup and easy bleeding, measurement of pocket depth and teeth X-rays by oral panoramic radiograph using Promax 3D (Planmeca OY; tube type D-054SB-C, 84 kV, 16 mA, filtration $2,5 \mathrm{~mm} \mathrm{AI}$ ). The exclusion criteria included periodontal treatment in the past six months, a history of diabetes mellitus, metabolic syndrome, other endocrine diseases, cardiovascular and cerebrovascular diseases, rheumatic diseases, chronic renal failure, nephrotic syndrome, obesity [body mass index $\left.(B M I) \geq 30 \mathrm{~kg} / \mathrm{m}^{2}\right]$, recent diagnosis with an infectious disease, pregnancy, lactation, hormone replacement therapy, chronic treatment with non-steroidal anti-inflammatory drugs or bisphosphonate or glucocorticoids, smokers and ex-smokers. All subjects were divided into the normal group (NC-h) and the hyperlipidemia group (HC-h). Their body weights $(\mathrm{kg})$ and heights $(\mathrm{m})$ were measured to calculate body mass index (BMI) as $\left(\mathrm{kg} / \mathrm{m}^{2}\right)$. Fasting peripheral venous blood samples were collected into anticoagulant tubes and centrifuged at 2,200 x g for $5 \mathrm{~min}$ at $25^{\circ} \mathrm{C}$ to prepare individuals serum samples. The levels of serum triglycerides (TG; $\mathrm{mg} / \mathrm{dl}$ ), total cholesterol (TC; mg/dl), high-density lipoprotein (HDL; mg/dl) and low-density lipoprotein (LDL; mg/dl) were measured using
Biobase reagent kit (BIOBASE LLC) in an autobiochemical analyzer (cat. no. AU5400; Olympus Corporation). Patients were diagnosed with hyperlipidemia, based on their serum levels of TC $>200 \mathrm{mg} / \mathrm{dl}$, TG $>200 \mathrm{mg} / \mathrm{dl}$, LDL-C $>130 \mathrm{mg} / \mathrm{dl}$ and HDL-C $<35 \mathrm{mg} / \mathrm{dl}$.

Periodontal examination. Periodontal examination was performed by an experienced dentist. All teeth in the mouth of individual patients were examined for their looseness (L), sulcus bleeding index (SBI), clinical attachment level (CAL), probing depth (PD) at six sites and the distance from the gingival margin to the cementoenamel junction (CEJ) at six sites (mesiobuccal, mid buccal, distobuccal, distolingual, mid lingual and mesiolingual). In addition, missing teeth were recorded (not including the third molar). The distance between the near and middle CEJ-alveolar bone crest $(\mathrm{ABC})$ of each tooth was measured three times using Planmeca Romexis 3.0.1.R software (Planmeca OY; accurate to $0.1 \mathrm{~mm})$.

Animal experiments. The animal experimental study was approved by The Animal Ethics Committee of the Shandong Provincial Hospital (approval no.2016-06) and the experimental procedures were in accordance with the Guide for the Care and Use of Laboratory Animals (12). Male Sprague-Dawley (SD) rats ( $\mathrm{n}=30$; age, 6 weeks; weight, 170-190 g) were obtained from Beijing Vital River Laboratory Animal Technology Co., Ltd. Animals were housed in a specific pathogen-free room under a consistent temperature $\left(23 \pm 2^{\circ} \mathrm{C}\right)$ and humidity $(55 \pm 10 \%)$ with a cycle of 12-h light/dark and free access to water and chow. The rats were randomized and fed with standard rodent chow [normal control (NC) group; $n=10]$, or high cholesterol rodent chow (Table I, Beijing Vital River Laboratory Animal Technology Co., Ltd.) containing $2 \%$ cholesterol $(n=20)$ for 32 weeks. Beginning from the 25 th week of a high cholesterol diet, the high cholesterol-fed rats were randomized to treatment with vehicle saline [hypercholesterolemia (HC) group; $\mathrm{n}=10$ ] or with $5 \mathrm{mg} / \mathrm{kg}$ simvastatin by gavage daily for 8 weeks [simvastatin (SIM) group; $n=10$ ]. During the last 8-week period, the NC rats were treated with vehicle saline. Their body weights were measured weekly. At the end of the experiment, abdominal aorta blood samples $(5-10 \mathrm{ml})$ were obtained from individual rats and their serum samples were prepared. The rats were euthanized following excessive anesthesia with $200 \mathrm{mg} / \mathrm{kg}$ pentobarbital sodium.

Laboratory examinations. The levels of serum TG, TC, HDL and LDL in individual rats were determined by enzymatic methods in an autobiochemical analyzer (cat. no. AU5400; Olympus).

Analysis of alveolar bone resorption. The maxillae from individual rats were dissected and separated as right and left sides. The semi-maxillary bone was stained with $1 \%$ methylene blue dye at $25^{\circ} \mathrm{C}$ for $1 \mathrm{~min}$ to demarcate the cement-enamel junction (CEJ). Both buccal and lingual root surfaces of all molar teeth were imaged using a digital camera (Canon). Alveolar bone loss was evaluated using Image-Pro Plus version 6.0 (Media Cybernetics, Inc.) in a blinded manner and was expressed in $\mathrm{mm}$. The mean of 3 buccal and 3 lingual surfaces on each 
Table I. Composition of the high cholesterol diet.

\begin{tabular}{lcc}
\hline Component & $\begin{array}{c}\text { Normal } \\
\text { control diet }\end{array}$ & $\begin{array}{c}\text { High } \\
\text { cholesterol diet }\end{array}$ \\
\hline Protein $(\mathrm{g} / 100 \mathrm{~g})$ & 20 & 17 \\
Carbohydrate $(\mathrm{g} / 100 \mathrm{~g})$ & 58 & 49 \\
Fat $(\mathrm{g} / 100 \mathrm{~g})$ & 6 & 21 \\
Selenium $(\mathrm{g} / 100 \mathrm{~g})$ & $1.4 \times 10^{-5}$ & $1.6 \times 10^{-5}$ \\
Cholesterol $(\mathrm{g} / 100 \mathrm{~g})$ & 0 & 2 \\
Fatty acids $(\mathrm{g} / 100 \mathrm{~g})$ & & \\
$\mathrm{C} 14: 0$ & 0.02 & 0.02 \\
$\mathrm{C} 16: 0$ & 0.97 & 0.97 \\
C16:1 & 0.02 & 0.02 \\
$\mathrm{C} 18: 0$ & 0.21 & 0.21 \\
C18:1 & 1.23 & 1.23 \\
C18:2 & 2.57 & 2.57 \\
C18:3 & 0.17 & 0.17 \\
Total saturated & 1.11 & 1.11 \\
Total monounsaturated & 1.22 & 1.22 \\
Total polyunsaturated & 2.93 & 2.93 \\
Total kcal/g & 3.4 & 3.4 \\
\hline
\end{tabular}

molar was calculated as the total alveolar bone loss in each animal.

Histology. The semi-maxillae were fixed in $4 \%$ paraformaldehyde at $25^{\circ} \mathrm{C}$ for $48 \mathrm{~h}$ and decalcified in $10 \%$ EDTA solution (pH 7.2) at $25^{\circ} \mathrm{C}$ for 2 weeks, followed by paraffin-embedding. The bone longitudinal sections $(5 \mu \mathrm{m})$ were stained with hematoxylin and eosin (H\&E). From each section 3-5 fields were randomly selected and imaged under an Olympus light microscope (model BX51TRF; Olympus Corporation).

Immunohistochemistry. The expression levels of $\mathrm{NF}-\kappa \mathrm{B}$, LC3 and p62 proteins in the bone tissues were determined by immunohistochemistry. The bone tissue sections $(4 \mu \mathrm{m})$ were dewaxed in xylene I and II solution at $25^{\circ} \mathrm{C}$ for $10 \mathrm{~min}$ each and rehydrated by serial decreased concentrations of ethanol [100 (I), 100 (II), 95, 80 and 70\%] for 5 min each. The tissue sections were subjected to antigen retrieval in $0.125 \%$ pancreatin at $37^{\circ} \mathrm{C}$ for $30 \mathrm{~min}$ and treated with $3 \% \mathrm{H}_{2} \mathrm{O}_{2}$ in methanol at $25^{\circ} \mathrm{C}$ for $30 \mathrm{~min}$. The tissue sections were blocked with 3\% bovine serum albumin (Shanghai Yeasen Biotechnology Co., Ltd.; cat. no. 36104ES25) in TBST at $25^{\circ} \mathrm{C}$ for $1 \mathrm{~h}$ and incubated with rabbit anti-NF- $\mathrm{B}$ p65 (1:200; cat. no. ab16502), anti-LC3B (1:100; cat. no. ab63817), anti-p62 (1:200; cat. no. ab91526; all, Abcam) at $4^{\circ} \mathrm{C}$ overnight. After washing with PBS, the sections were incubated with biotinylated goat anti-rabbit IgG (1:400; cat. no. ab150077; Abcam). Bound antibodies were detected with peroxidase-labeled streptavidin (Shanghai Herochem Co., Ltd.) at $37^{\circ} \mathrm{C}$ for 30 min, followed by development with Diaminobenzidine (OriGene Technologies, Inc.) and counterstained with haematoxylin at $25^{\circ} \mathrm{C}$ for $1 \mathrm{~min}$. The stained signals in six fields of view were imaged under a light microscope (Olympus CX21; Olympus Corporation). The stained intensity of density (IOD) and the ratio of the stained areas to total areas (sum/area sum) in each image were analyzed using Image-Pro Plus software version 6.0 (Media Cybernetics, Inc.) in a blinded manner, as described previously (13).

Reverse transcription-quantitative PCR. The levels of OPG, RANKL, NF- $\kappa$ B, LC3 and p62 mRNA transcripts, relative to the control GAPDH, were determined in the bone tissues by RT-qPCR. Briefly, total RNA was extracted from each alveolar bone tissue using Trizol ${ }^{\circledR}$ reagent (Thermo Fisher Scientific, Inc.) and reverse transcribed into cDNA using the PrimeScript ${ }^{\mathrm{TM}}$ RT reagent kit (Takara Bio., Inc.) according to the manufacturer's protocol. Subsequently, qPCR was performed using the FastStart DNA Master SYBR Green I kit (Takara Bio) with specific primers in a LightCycler System 480 (Roche Diagnostics). The PCR reactions were performed in duplicate at $95^{\circ} \mathrm{C}$ for $30 \mathrm{sec}$ and subjected to 40 cycles of $95^{\circ} \mathrm{C}$ for $5 \mathrm{sec}$ and $60^{\circ} \mathrm{C}$ for $30 \mathrm{sec}$. Data were analyzed using the $2^{-\Delta \Delta \mathrm{Cq}}$ method (14). The primer sequences were forward 5'CCC TCTCTCTGCTCACTCTGCT3' and reverse 5'CTTACTGCC CTCCTGCTTGG3' for GAPDH; forward 5'TCAGTTCCA TGGCCCAGAC3' and reverse 5'GTTGTCTTTGAGATCCAT GCCATT3' for TNF- $\alpha$; forward 5'CCCTGAACTCAACTG TGAAATAGCA3' and reverse 5'CCCAAGTCAAGGGCT TGGAA3' for IL-1 $\beta$; forward 5'TGTGGAATAGATGTCACC CTGTGC3' and reverse 5'CACAGAGGTCAATGTCTTGGA TGATC3' for OPG; forward 5'GCTTCTCAGGAGTTCCAG CTATGAT3' and reverse 5'CGTTGCTTAACGTCATGTTAG AGATCT3' for RANKL; forward 5'GCTATAATCCTGGAC TTCTG3' and reverse 5'GAGGAAGGCTGTGAACATGA3' for NF- $\kappa \mathrm{B}$; forward 5'GAGTGGAAGATGTCCGGCTC3' and reverse 5'CCAGGAGGAAGAAGGCTTGG3' for LC3; and forward 5'GCTGCTCTCTTCAGGCTTACAG3' and reverse 5'CCTGCTTCACAGTAGACGAAAG3' for p62.

Statistical analysis. All experiments were independently performed in triplicate. Data are presented as the mean \pm SD. The difference between two experimental groups was analyzed by the unpaired Student's t-test using the SPSS, version 17.0 (SPSS, Inc.). The correlation between blood lipid concentrations and periodontal index was analyzed by Spearman's rank correlation analysis. $\mathrm{P}<0.05$ was considered statistically significant.

\section{Results}

Hyperlipidemia is associated with severe alveolar bone resorption in patients with periodontitis. To determine the potential effect of hyperlipidemia on alveolar bone loss, 100 patients with periodontitis were recruited and stratified into the hyperlipidemia and normal lipid level groups, according to their serum TC, TG, LDL-C and HDL-C levels. Their demographic and laboratory characteristics are reported in Table II. The levels of serum TC, TG and LDL-C in the hyperlipidemia group were significantly higher than that in the normal lipid group, while the serum HDL-C levels were significantly lower in the hyperlipidemia group than in the normal lipid group. Clinically, periodontal examination revealed that the levels of SBI, CAL, PD and CEJ-ABC, but not tooth looseness (L), in the hyperlipidemia group were significantly higher than that in 
Table II. Characteristics of the study population (mean \pm standard deviation).

\begin{tabular}{lccr}
\hline Characteristic & NC-h $(\mathrm{n}=52)$ & HC-h $(\mathrm{n}=48)$ & P-value \\
\hline Age (years) & $42.56 \pm 10.39$ & $44.12 \pm 9.87$ & 0.348 \\
Male/female & $28 / 24$ & $27 / 21$ & 0.164 \\
BMI $\left(\mathrm{kg} / \mathrm{m}^{2}\right)$ & $24.73 \pm 2.5$ & $26.12 \pm 3.4$ & 0.083 \\
TC $(\mathrm{mg} / \mathrm{dl})$ & $135.92 \pm 14.07$ & $242.41 \pm 17.89$ & $<0.001$ \\
TG $(\mathrm{mg} / \mathrm{dl})$ & $127.13 \pm 16.54$ & $248.70 \pm 16.25$ & $<0.001$ \\
LDL-C $(\mathrm{mg} / \mathrm{dl})$ & $81.24 \pm 12.09$ & $167.95 \pm 13.13$ & $<0.001$ \\
HDL-C $(\mathrm{mg} / \mathrm{dl})$ & $55.79 \pm 6.29$ & $21.87 \pm 3.21$ & $<0.001$ \\
L & $0.19 \pm 0.15$ & $0.26 \pm 0.21$ & 0.449 \\
SBI & $0.94 \pm 0.37$ & $2.59 \pm 0.42$ & $<0.001$ \\
CAL $(\mathrm{mm})$ & $0.58 \pm 0.46$ & $2.72 \pm 0.89$ & $<0.001$ \\
PD $(\mathrm{mm})$ & $1.29 \pm 0.56$ & $3.24 \pm 0.75$ & $<0.001$ \\
CEJ-ABC $(\mathrm{mm})$ & $1.09 \pm 0.80$ & $2.32 \pm 0.81$ & $<0.001$
\end{tabular}

BMI, body mass index; TC, total cholesterol; TG, plasma triglyceride; LDL-C, low density lipoprotein cholesterol; HDL-C, high density lipoprotein cholesterol; L, Tooth looseness; SBI, sulcus bleeding index; CAL, attachment loss; PD, probing depth; CEJ-ABC, cemantoenamel junction-alveolar bone crest.

the normal lipid level group. The levels of TC, TG and LDL-C were positively correlated with the values of SBI, CAL, PD, and CEJ-ABC, while the levels of HDL-C were negatively correlated with the values of SBI, CAL, PD and CEJ-ABC in this population (Table III). These data indicated that hyperlipidemia was correlated positively with the severity of alveolar bone loss during the process of periodontitis in humans.

Simvastatin intervention reduces hypercholesterolemia and its-related alveolar bone resorption in rats. Next, we tested whether treatment with simvastatin could mitigate the hypercholesterolemia-related alveolar bone resorption in rats. After fed with high cholesterol food for 24 weeks, the high cholesterol-fed rats were randomized and treated with vehicle saline (HC group) or simvastatin (SIM group) by gavage daily for 8 weeks under continual high cholesterol diet. Another group of rats were fed with normal chow and received vehicle treatment as the normal control (NC). In comparison with the NC, although we observed gradually increased body weights with time in the high cholesterol-fed rats there was no significant difference among the groups ( $\mathrm{P}>0.05$, Fig. 1A). As expected, we detected significantly increased levels of serum TC, and LDL-C, but not TG and HDL-C in the high cholesterol-fed rats $(\mathrm{P}<0.001$ for both, Fig. 1B). The levels of serum TC, and LDL-C in the SIM group were significantly lower than that in the HC group $(\mathrm{P}<0.001, \mathrm{P}<0.05)$.

Next, we measured the linear distance between the CEJ and $\mathrm{ABC}$ to evaluate the levels of alveolar bone loss in individual groups of rats (Fig. $1 \mathrm{C}$ and D). We found that the linear distance between the CEJ and $\mathrm{ABC}$ in the SIM group of rats was significantly shorter than that in the $\mathrm{HC}$ group $(\mathrm{P}<0.05)$, but remained significantly longer than that in the NC group of rats $(\mathrm{P}<0.05$, Fig. $1 C)$. Hence, such data indicated that high cholesterol feeding induced hypercholesterolemia and alveolar bone loss, where were significantly mitigated by simvastatin treatment in rats.
Simvastatin intervention mitigates hypercholesterolemia-modulated. OPG, RANKL and NF- $\mathrm{B}$ expression in the alveolar bone of rats. Given that OPG and RANKL are crucial for osteoclastogenesis and hyperlipidemia can activate the NF- $\kappa$ B signaling though engagement of TLR2/4 (15), the potential mechanisms underlying the action of simvastatin in regulating hypercholesterolemia-induced alveolar bone loss in rats were explored. The relative levels of NF- $\mathrm{NB}$ expression in the alveolar bone tissues of the different groups of rats were measured. Levels of $\mathrm{NF}-\kappa \mathrm{B}$ mRNA transcripts in the SIM group were significantly lower than those in the HC group $(\mathrm{P}<0.05)$, but still significantly higher than those in the NC group of rats $(\mathrm{P}<0.001$; Fig. $2 \mathrm{~A})$. Further immunohistochemistry revealed anti-NF- $\kappa \mathrm{B}+$ granules in the nucleus and cytoplasm of osteoclast-like cells and that the intensity of anti-NF- $\kappa$ Bp65 staining in in the SIM group was obviously lower than in the $\mathrm{HC}$ group, but still higher than that in the NC group (Fig. 2B and C). Although a significant difference in the relative levels of TNF- $\alpha$ and IL-1 $\beta$ mRNA transcripts in the alveolar bone tissues from different groups of rats was not detected ( $\mathrm{P}>0.05$ for all; Fig. 3A), significantly higher levels of RANKL and OPG mRNA transcripts, leading to high ratios of RANKL to OPG in the HC group relative to the NC group were observed (Fig. 3B). However, simvastatin treatment significantly reduced the levels of RANKL, but not OPG, mRNA transcripts, decreasing the ratios of RANKL to OPG mRNA transcripts in rats. Collectively, such data indicated that simvastatin intervention minimized hypercholesterolemia-enhanced NF- $\kappa$ B expression and modulated RANKL and OPG transcription, ameliorating hypercholesterolemia-related alveolar bone loss in rats.

Simvastatin intervention modulates LC3 and p62 expression in the alveolar bone of hypercholesterolemia rats. Hyperlipidemia can enhance oxidative stress and increase autophagy during the process of osteoclastogenesis. The 

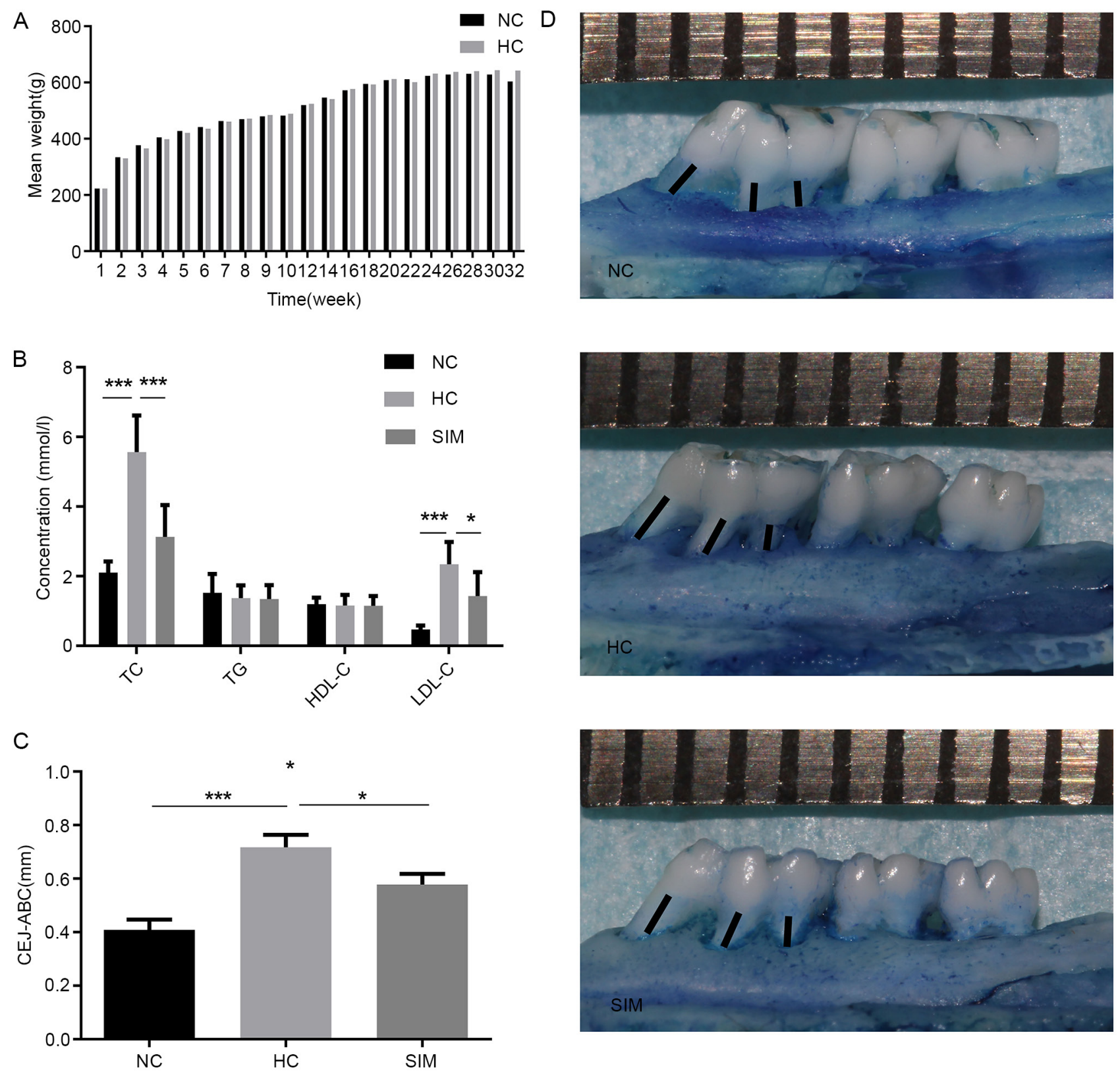

Figure 1. Simvastatin reduces the hypercholesterolemia-induced alveolar bone loss in rats. Rats were fed with normal rat chow for 32 weeks or with chow containing $2 \%$ cholesterol for 32 weeks. Beginning at the 25 th weeks of high cholesterol diet, the high cholesterol-fed rats were randomized, continually fed with high cholesterol diet, and treated with vehicle saline or simvastatin by gavage daily for 8 weeks. (A) Rat body weights were measured weekly and (B) their TG, TC, HDL and low-density LDL levels were quantified. (C) The linear distance between the cement-enamel CEJ and the ABC was measured in (D) images of the buccal and lingual root surfaces. Each small grid on the upper ruler represents $1 \mathrm{~mm}$. The black lines represent the distance of alveolar bone resorption. Results are presented as the mean $\pm \mathrm{SD}$ of each group ( $\mathrm{n}=10$ per group). ${ }^{*} \mathrm{P}<0.05 ;{ }^{* * *} \mathrm{P}<0.001$. NC, normal control group; HC, hypercholesterolemia group; SIM, simvastatin group; TC, total cholesterol; TG, plasma triglyceride; LDL, low density lipoprotein; HDL, high density lipoprotein; CEJ, cement-enamel junction; $\mathrm{ABC}$, alveolar bone crest.

possible effect of simvastatin intervention on hypercholesterolemia-enhanced LC3 and p62 expression in the alveolar bone tissues was assessed by RT-qPCR and immunohistochemistry. Compared with the NC group, significantly increased levels of LC3 and p62 mRNA transcripts were detected in the alveolar bone tissues of the $\mathrm{HC}$ group $(\mathrm{P}<0.01, \mathrm{P}<0.05$, Fig. 4A). Simvastatin intervention did not significantly change the relative levels of LC3 mRNA transcripts, but did significantly reduce the levels of p62 mRNA transcripts in the alveolar bone tissues of the SIM group of rats, relative to that the $\mathrm{HC}$ group $(\mathrm{P}<0.01$ Fig. $4 \mathrm{~A}$ and $\mathrm{B})$. As a result, simvastatin intervention increased the ratios of LC3 to p62 mRNA transcripts in the alveolar bone tissues of the SIM group of rats $(\mathrm{P}<0.05$ Fig. 4C). Immunohistochemistry revealed that the intensity of anti-LC3 staining in the SIM group was similar to that in the $\mathrm{NC}$ group, but less than that in the $\mathrm{HC}$ group of rats (Fig. 4D-F). A similar pattern of anti-p62 staining was observed in the alveolar bone tissues from the different groups of rats (Fig. 4G-I). Simvastatin intervention may therefore modulate LC3 and p62 expression and increase the ratios of LC3 to p62 expression in the alveolar bone tissues of hypercholesterolemia rats. 
Table III. Partial correlation after controlling for confounding variables.

\begin{tabular}{|c|c|c|c|c|c|c|c|c|}
\hline & $\mathrm{TC}$ & TG & LDL-C & HDL-C & SBI & CAL & $\mathrm{PD}$ & CEJ-ABC \\
\hline \multicolumn{9}{|l|}{$\mathrm{TC}$} \\
\hline Correlation & 1 & $0.752^{\mathrm{a}}$ & $0.702^{a}$ & $-0.909^{a}$ & $0.490^{\mathrm{a}}$ & $0.560^{\mathrm{a}}$ & $0.489^{a}$ & $0.525^{\mathrm{a}}$ \\
\hline Significance (two-tailed) & & $<0.001$ & $<0.001$ & $<0.001$ & $<0.001$ & $<0.001$ & $<0.001$ & $<0.001$ \\
\hline \multicolumn{9}{|l|}{ TG } \\
\hline Correlation & & 1 & $0.762^{\mathrm{a}}$ & $-0.712^{\mathrm{a}}$ & $0.595^{\mathrm{a}}$ & $0.591^{\mathrm{a}}$ & $0.445^{\mathrm{a}}$ & $0.593^{\mathrm{a}}$ \\
\hline Significance (two-tailed) & & & $<0.001$ & $<0.001$ & $<0.001$ & $<0.001$ & $<0.001$ & $<0.001$ \\
\hline \multicolumn{9}{|l|}{ LDL-C } \\
\hline Correlation & & & 1 & $-0.761^{\mathrm{a}}$ & $0.529^{a}$ & $0.557^{\mathrm{a}}$ & $0.439^{\mathrm{a}}$ & $0.587^{\mathrm{a}}$ \\
\hline Significance (two-tailed) & & & & $<0.001$ & $<0.001$ & $<0.001$ & $<0.001$ & $<0.001$ \\
\hline \multicolumn{9}{|l|}{ HDL-C } \\
\hline Correlation & & & & 1 & $-0.484^{\mathrm{a}}$ & $-0.561^{\mathrm{a}}$ & $-0.429^{a}$ & $-0.562^{\mathrm{a}}$ \\
\hline Significance (two-tailed) & & & & & $<0.001$ & $<0.001$ & $<0.001$ & $<0.001$ \\
\hline \multicolumn{9}{|l|}{ SBI } \\
\hline Correlation & & & & & 1 & $0.360^{\mathrm{a}}$ & $0.250^{\mathrm{a}}$ & $0.414^{\mathrm{a}}$ \\
\hline Significance (two-tailed) & & & & & & $<0.001$ & 0.012 & $<0.001$ \\
\hline \multicolumn{9}{|l|}{ CAL } \\
\hline Correlation & & & & & & 1 & $0.333^{\mathrm{a}}$ & $0.550^{\mathrm{a}}$ \\
\hline Significance (two-tailed) & & & & & & & 0.001 & $<0.001$ \\
\hline \multicolumn{9}{|l|}{ PD } \\
\hline Correlation & & & & & & & 1 & $0.357^{\mathrm{a}}$ \\
\hline Significance (two-tailed) & & & & & & & & $<0.001$ \\
\hline \multicolumn{9}{|l|}{ CEJ-ABC } \\
\hline Correlation & & & & & & & & 1 \\
\hline Significance (two-tailed) & & & & & & & & \\
\hline
\end{tabular}

TC, total cholesterol; TG, plasma triglyceride; LDL-C, low density lipoprotein cholesterol; HDL-C, high density lipoprotein cholesterol; L, Tooth looseness; SBI, sulcus bleeding index; CAL, attachment loss; PD, probing depth; CEJ-ABC, cemantoenamel junction-alveolar bone crest. ${ }^{\mathrm{a}} \mathrm{P} \leq 0.01$.

\section{Discussion}

A previous study demonstrated a positive correlation between deep periodontal pockets and elevated blood lipid levels (16). In the present study, patients with periodontitis and hyperlipidemia displayed severe periodontal tissue damage and alveolar bone resorption in comparison to those with normal lipid levels, consistent with previous observations (16). The level of hyperlipidemia was positively correlated with the severity of periodontal tissue damage in this population, supporting the hypothesis that hyperlipidemia is associated with the progression of periodontitis-related alveolar bone loss (17). Hyperlipidemia can increase innate immune cell activity (18) and activate innate immune cells, including macrophages and natural killer cells, which can secrete pro-inflammatory cytokines and migrate into the inflammatory lesion, worsening periodontitis and promoting alveolar bone resorption (19). Assessing the degree of hyperlipidemia may therefore be beneficial when evaluating the severity of periodontitis.

Simvastatin can promote bone formation and increase $\mathrm{BMD}$ in rats with hyperlipidemia, and ameliorate hypercholesterolemia-related microstructure changes in the jaw bone of animals (10). A study suggested that simvastatin may promote bone formation and reduce alveolar bone loss in the maxillary following ovariectomy and ligature placement in rats (20). To understand the therapeutic effect and potential mechanisms underlying the action of simvastatin, a rat model of hypercholesterolemia-related periodontitis was established in the present study. High cholesterol feeding was demonstrated not only to induce hypercholesterolemia, but also to promote severe alveolar bone loss in rats. By contrast, treatment with simvastatin did not significantly alter rat body weights, but significantly ameliorated hypercholesterolemia and alveolar bone loss, extending previous observations $(10,20)$. These findings suggest that treatment with simvastatin may benefit patients with periodontitis by preventing and inhibiting alveolar bone loss.

The RANKL/RANK/OPG system is crucial for bone metabolism and its imbalance is associated with promoting osteoclastogenesis by activating $\mathrm{c}-\mathrm{Fos}, \mathrm{NF}-\kappa \mathrm{B}$ and nuclear factor of activated $\mathrm{T}$ cells 1 (21). $\mathrm{NF}-\kappa \mathrm{B}$ activation can promote the early differentiation of osteoclast precursors, while inhibition of $N F-\kappa B$ activation prevents RANKL and 


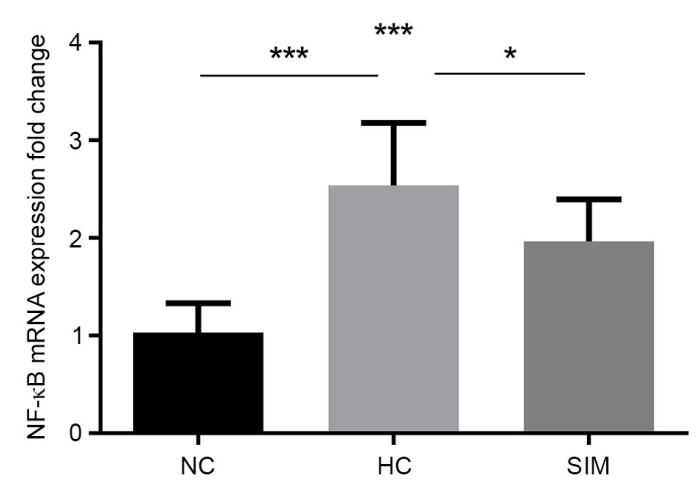

B

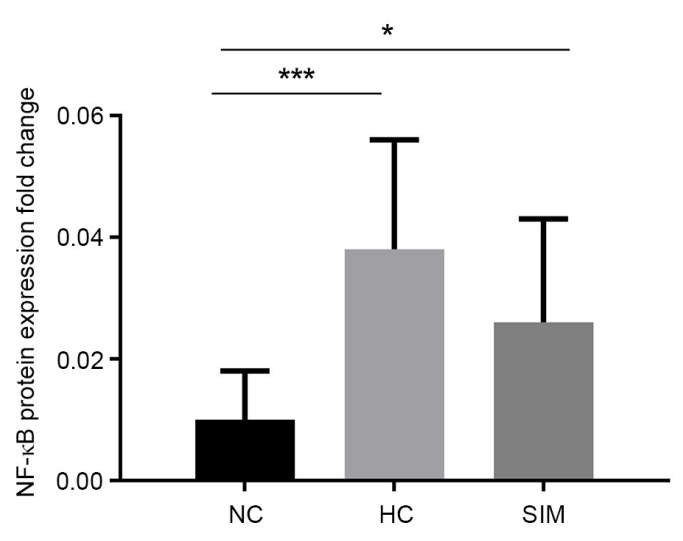

C
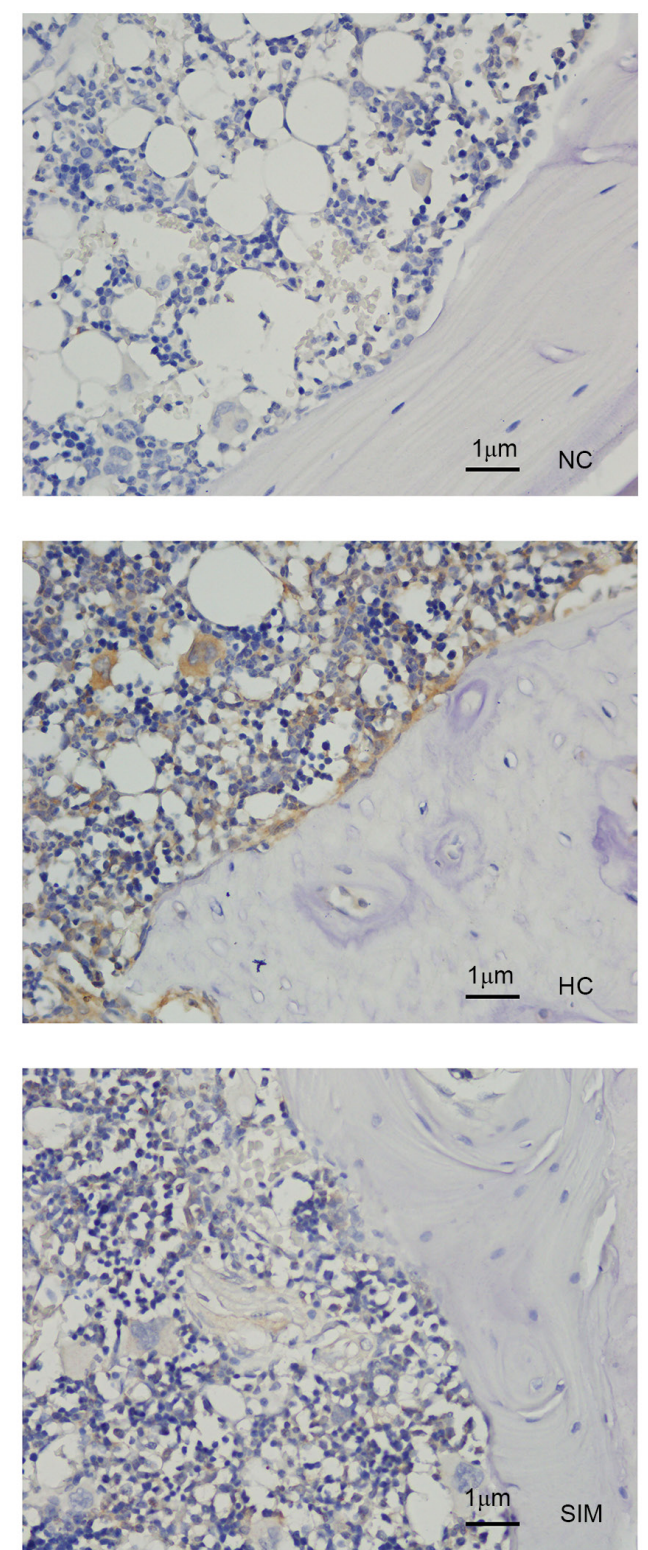

Figure 2. Simvastatin reduces hypercholesterolaemia-enhanced NF- $\kappa$ B expression in the alveolar bone tissues of rats. The levels of NF- $\kappa$ B mRNA transcripts and protein expression in the alveolar bone tissues of the different groups of rats were quantified by reverse transcription-quantitative PCR and immunohistochemistry. (A) The relative levels of NF- $\kappa$ B mRNA transcripts. (B) Immunohistochemistry of NF- $\kappa$ B p65 protein expression and (C) quantification. Data are representative images (magnification, $\mathrm{x} 200)$ or presented as the mean $\pm \mathrm{SD}$ of each group $(\mathrm{n}=10)$ from three separate experiments $\mathrm{NC}$, normal control group; $\mathrm{HC}$, hypercholesterolemia group; SIM, simvastatin group; $\mathrm{NF}-\kappa \mathrm{B}$, nuclear factor- $\kappa \mathrm{B} .{ }^{*} \mathrm{P}<0.05 ;{ }^{* * * *} \mathrm{P}<0.001$.

TNF $\alpha$-induced bone resorption (22). NF- $\kappa \mathrm{B}$ signaling is also involved in the progression of periodontitis, damaging the alveolar bone and periodontal ligament $(23,24)$. In the present study, simvastatin intervention significantly mitigated hypercholesterolemia-induced $\mathrm{NF}-\kappa \mathrm{B}$ and RANKL expression, but not that of OPG, TNF- $\alpha$ and IL-1 $\beta$, in the alveolar bone tissues of rats, consistent with a previous report $(25,26)$. These findings extended previous observations that simvastatin inhibits $N F-\kappa B$ signaling and RANKL-induced osteoclastogenesis in RAW 264.7 cells and alveolar bone loss induced by lipopolysaccharide in rats $(27,28)$. Inhibition of $N F-\kappa B$ signaling and related RANKL expression by simvastatin may therefore be crucial for the control of hypercholesterolemia-related periodontitis and alveolar bone loss in rats.
Autophagy can regulate bone resorption by osteoclasts (7). In the present study, while hypercholesterolemia enhanced LC3 and p62 expression, simvastatin intervention did not significantly alter the levels of LC3 expression, but significantly reduced the levels of p62 expression, leading to an increase in the ratios of LC3 to p62 expression in the alveolar bone tissues of rats. It may be hypothesized that increased ratios of LC3 to p62 expression predominantly occur in osteoclasts and enhance their autophagic flux, which may contribute to the simvastatin-induced inhibition of alveolar bone loss in rats (29). Given that p62 is the most specific adaptor protein to regulate the formation of protein aggregates and is continuously degraded by the autophagolysosome $(30,31)$ the increased ratios of LC3 to p62 expression by simvastatin may at least partially explain 

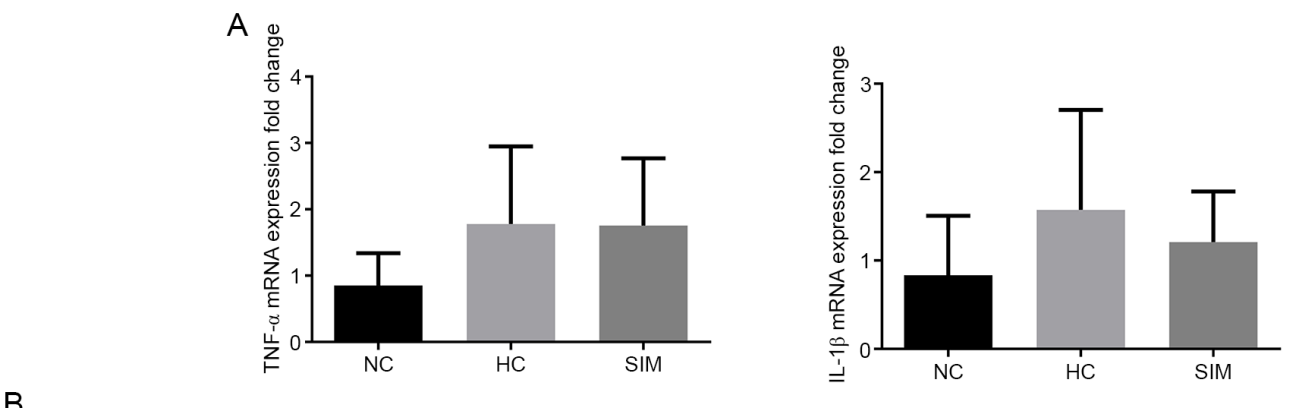

B
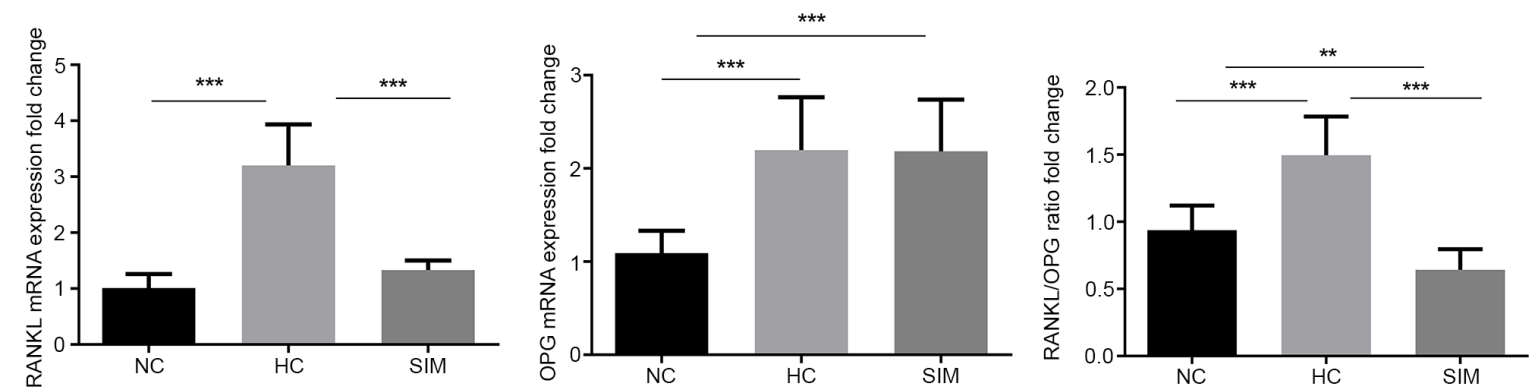

Figure 3. Simvastatin modulates the ratios of RANKL to OPG expression in the alveolar bone of rats. The relative levels of TNF- $\alpha$, IL-1 $\beta$, RANKL and OPG mRNA transcripts in the alveolar bone of the different groups of rats were quantified by reverse transcription-quantitative PCR and the ratios of RANKL to OPG expression were calculated. Data are presented as the mean \pm SD of each group $(n=10)$ from three separate experiments. (A) The relative levels of TNF- $\alpha$ and IL-1 $\beta$ mRNA transcripts. (B) The relative levels of RANKL and OPG mRNA transcripts and the ratios of RANKL to OPG in the different groups of rats. NC, normal control group; HC, hypercholesterolemia group; SIM, simvastatin group; TNF- $\alpha$, tumor necrosis factor- $\alpha$; IL-1 $\beta$, interleukin-1 $\beta$; RANKL, receptor activator of nuclear factor-kB ligand; OPG, osteoprotegerin. ${ }^{* *} \mathrm{P}<0.01 ;{ }^{* * *} \mathrm{P}<0.001$.
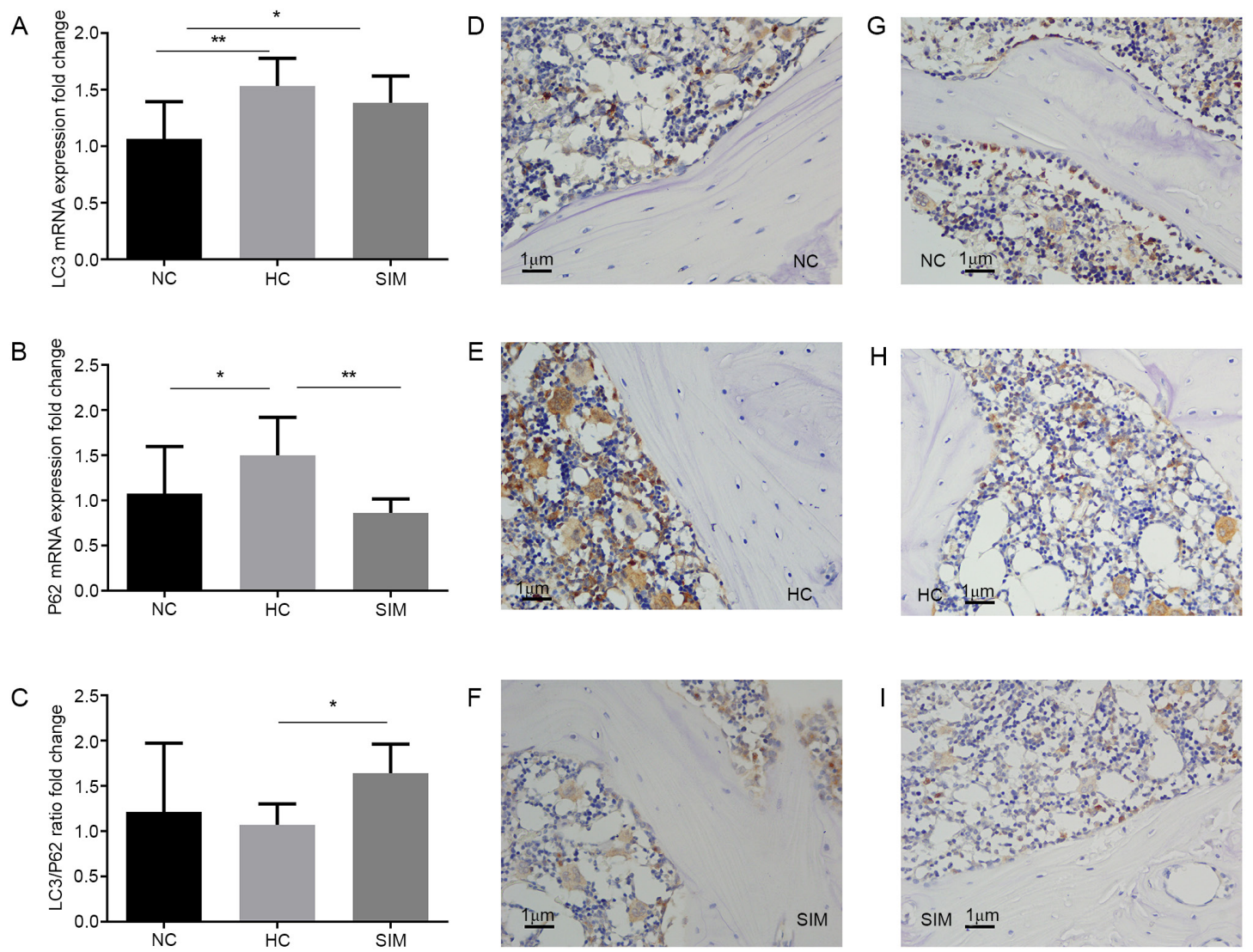

Figure 4. Simvastatin increases the ratios of LC3 to p62 expression in the alveolar bone of rats. The relative levels of LC3 and p62 expression in the alveolar bone tissues of the different groups of rats were examined by reverse transcription-quantitative PCR and immunohistochemistry and the ratios of LC3 to p62 mRNA transcripts were calculated. Results are representative images (magnification, $x 200$ ) or presented as the mean \pm SD of each group ( $\mathrm{n}=10$ ) from three separate experiments. The relative levels of (A) LC3 and (B) p62 mRNA transcripts and (C) their ratios. Immunohistochemistry analysis of LC3 expression in the alveolar bone tissues of the (D) NC, (E) HC and (F) SIM group. Immunohistochemistry analysis of p62 expression in the alveolar bone tissues of the (G) NC, (H) HC and (I) SIM group. NC, normal control group; HC, hypercholesterolemia group; SIM, simvastatin group; LC3, microtubule-associated protein 1 light chain. ${ }^{*} \mathrm{P}<0.05,{ }^{* *} \mathrm{P}<0.01$. 
its therapeutic role in the inhibition of hypercholesterolemia-mediated bone loss in the alveolar bone of rats (32-34). Modulation of autophagy may therefore be a viable strategy for the inhibition of hypercholesterolemia-related alveolar bone loss.

The present study had numerous limitations, including a relatively small sample size for a human study without prospective simvastatin treatment; measuring cytokine expression at the level of mRNA transcripts and not their proteins; assessing $\mathrm{NF}-\kappa \mathrm{B}, \mathrm{p} 62$ expression but not its phosphorylation level; measuring LC3 but not LC3 I and II expression; the lack of specific markers for identification of osteoclasts in bone tissues; and the lack of more valuable measures for determining autophagy. Hence, further investigation of the therapeutic effect and potential mechanisms underlying the action of simvastatin in regulating hyperlipidemia-related alveolar bone loss in a larger population is warranted.

In conclusion, the present results indicated that hyperlipidemia was associated with alveolar bone loss in patients with periodontitis. Simvastatin intervention not only reduced hypercholesterolemia, but also mitigated hypercholesterolemia-related alveolar bone loss by reducing $N F-\kappa B$ and RANKL expression and modulating LC3 and p62 expression in the alveolar bone tissues of hypercholesterolemic rats. These data may suggest new mechanisms underlying the therapeutic action of simvastatin in intervention for periodontitis-related bone loss.

\section{Acknowledgements}

Not applicable.

\section{Funding}

The present study was supported by grants from the Shandong Provincial Natural Science Foundation, China (grant nos. 2016ZRB1462 and ZR2017BH005) and Shandong Provincial Health Commission Foundation (grant no. 2016WS0410).

\section{Availability of data and materials}

The datasets used and/or analyzed in the current study are available from the corresponding author on reasonable request.

\section{Authors' contributions}

$\mathrm{XG}, \mathrm{SH}$ and $\mathrm{DZ}$ participated the study design. $\mathrm{XG}$ and JZ performed the experiments. $X G$ and $Y B$ collected and analyzed the data. XG drafted the manuscript. XG and DZ confirmed the authenticity of all the raw data. All authors read and approved the final manuscript.

\section{Ethics approval and consent to participate}

The experimental protocols were approved by the Ethics Committee of Shandong Provincial Hospital (approval no. SWYX:NO.2021-013) and the Animal Ethics Committee of Shandong Provincial Hospital (approval no. 2016-06).
Written informed consent for participation was obtained from all patients.

\section{Patient consent for publication}

Not applicable.

\section{Competing interests}

The authors declare that they have no competing interests.

\section{References}

1. Acharya A, VanWormer JJ, Waring SC, Miller AW, Fuehrer JT and Nycz GR: Regional epidemiologic assessment of prevalent periodontitis using an electronic health record system. Am J Epidemiol 177: 700-707, 2013.

2. Kinane DF: Causation and pathogenesis of periodontal disease. Periodontol 2000 25: 8-20,2001.

3. Kaye EK: n-3 fatty acid intake and periodontal disease. J Am Diet Assoc 110: 1650-1652, 2010.

4. Macri E, Lifshitz F, Ramos C, Orzuza R, Costa O, Zago V, Boyer P and Friedman S: Atherogenic cholesterol-rich diet and periodontal disease. Arch Oral Biol 59: 679-686, 2014.

5. Li RF, Chen G, Ren JG, Zhang W, Wu ZX, Liu B, Zhao Y and Zhao YF: The adaptor protein $\mathrm{p} 62$ is involved in RANKL-induced autophagy and osteoclastogenesis. J Histochem Cytochem 62: 879-888, 2014.

6. Chung YH, Jang Y, Choi B, Song DH, Lee EJ, Kim SM, Song Y, Kang SW, Yoon SY and Chang EJ: Beclin-1 is required for RANKL-induced osteoclast differentiation. J Cell Physiol 229: 1963-1971, 2014.

7. DeSelm CJ, Miller BC, Zou W, Beatty WL, van Meel E, Takahata Y, Klumperman J, Tooze SA, Teitelbaum SL and Virgin HW: Autophagy proteins regulate the secretory component of osteoclastic bone resorption. Dev Cell 21: 966-974, 2011.

8. Towne SP and Thara E: Do statins reduce events in patients with metabolic syndrome? Curr Atheroscler Rep 10: 39-44, 2008.

9. Sadowitz B, Maier KG and Gahtan V: Basic science review: Statin therapy-Part I: The pleiotropic effects of statins in cardiovascular disease. Vasc Endovascular Surg 44: 241-251, 2010.

10. Zhou J, Gao X, Huang S, Ma L, Cui Y, Wang H, Qiu J, Wang L, Dong Q, Chen Z, et al: Simvastatin improves the jaw bone microstructural defect induced by high cholesterol diet in rats by regulating autophagic flux. Biomed Res Int 2018: 4147932, 2018.

11. Snaedal J: The Helsinki declaration. Laeknabladid 100: 135, 2014 (In Icelandic).

12. National Research Council (US) Committee for the Update of the Guide for the Care and Use of Laboratory Animals: Guide for the Care and use of Laboratory Animals. 8th edition. National Academies Press, Washington, DC, 2011.

13. Wang CJ, Zhou ZG, Holmqvist A, Zhang H, Li Y, Adell G and Sun XF: Survivin expression quantified by image pro-plus compared with visual assessment. Appl Immunohistochem Mol Morphol 17: 530-535, 2009.

14. Livak KJ and Schmittgen TD: Analysis of relative gene expression data using real-time quantitative PCR and the 2(-Delta Delta C(T)) method. Methods 25: 402-408, 2001.

15. Zhang P, Liu J, Xu Q, Harber G, Feng X, Michalek SM and Katz J: TLR2-dependent modulation of osteoclastogenesis by Porphyromonas gingivalis through differential induction of NFATc1 and NF-kappaB. J Biol Chem 286: 24159-24169, 2011.

16. Katz J, Flugelman MY, Goldberg A and Heft M: Association between periodontal pockets and elevated cholesterol and low density lipoprotein cholesterol levels. J Periodontol 73: 494-500, 2002.

17. Scardina GA, Pisano T, Cacioppo A and Messina P: Periodontal alteration of the microcirculation and hypercholesterolemia: A possible correlation? South Med J 104: 116-120, 2011. 
18. Croft KD, Beilin LJ, Vandongen R, Rouse I and Masarei J: Leukocyte and platelet function and eicosanoid production in subjects with hypercholesterolaemia. Atherosclerosis 83: 101-109, 1990.

19. Gustafsson A and Asman B: Increased release of free oxygen radicals from peripheral neutrophils in adult periodontitis after Fc delta-receptor stimulation. J Clin Periodontol 23: 38-44, 1996.

20. Xu XC, Chen H, Zhang X, Zhai ZJ, Liu XQ, Qin A and Lu EY: Simvastatin prevents alveolar bone loss in an experimental rat model of periodontitis after ovariectomy. J Transl Med 12: 284, 2014.

21. Wada T, Nakashima T, Hiroshi $\mathrm{N}$ and Penninger $\mathrm{JM}$ : RANKL-RANK signaling in osteoclastogenesis and bone disease. Trends Mol Med 12: 17-25, 2006.

22. Yamashita T, Yao Z, Li F, Zhang Q, Badell IR, Schwarz EM, Takeshita S, Wagner EF, Noda M, Matsuo K, et al: NF-kappaB p50 and p52 regulate receptor activator of NF-kappaB ligand (RANKL) and tumor necrosis factor-induced osteoclast precursor differentiation by activating c-Fos and NFATc1. J Biol Chem 282: 18245-18253, 2007.

23. Cochran DL: Inflammation and bone loss in periodontal disease J Periodontol 79 (Suppl 8): S1569-S1576, 2008.

24. Xu J, Wu HF, Ang ES, Yip K, Woloszyn M, Zheng MH and Tan RX: NF-kappaB modulators in osteolytic bone diseases. Cytokine Growth Factor Rev 20: 7-17, 2009.

25. Kaji H, Kanatani M, Sugimoto T and Chihara K: Statins modulate the levels of osteoprotegerin/receptor activator of NFkappaB ligand mRNA in mouse bone-cell cultures. Horm Metab Res 37: 589-592, 2005

26. Estanislau IM, Terceiro IR, Lisboa MR, Teles Pde B, Carvalho Rde S, Martins RS and Moreira MM: Pleiotropic effects of statins on the treatment of chronic periodontitis - a systematic review. Br J Clin Pharmacol 79: 877-885, 2015.

27. Ahn KS, Sethi G, Chaturvedi MM and Aggarwal BB: Simvastatin, 3-hydroxy-3-methylglutaryl coenzyme A reductase inhibitor, suppresses osteoclastogenesis induced by receptor activator of nuclear factor-kappaB ligand through modulation of NF-kappaB pathway. Int J Cancer 123: 1733-1740, 2008.
28. Jin J, Machado ER, Yu H, Zhang X, Lu Z, Li Y,Lopes-Virella MF, Kirkwood KL and Huang Y: Simvastatin inhibits LPS-induced alveolar bone loss during metabolic syndrome. J Dent Res 93: 294-299, 2014.

29. Maynard AA, Dvorak K, Khailova L, Dobrenen H, Arganbright KM, Halpern MD, Kurundkar AR, Maheshwari A and Dvorak B: Epidermal growth factor reduces autophagy in intestinal epithelium and in the rat model of necrotizing enterocolitis. Am J Physiol Gastrointest Liver Physiol 299: G614-G622, 2010.

30. Komatsu M, Kurokawa H, Waguri S, Taguchi K, Kobayashi A, Ichimura Y, Sou YS, Ueno I, Sakamoto A, Tong KI, et al: The selective autophagy substrate p62 activates the stress responsive transcription factor Nrf2 through inactivation of Keap1. Nat Cell Biol 12: 213-223, 2010.

31. Johansen T and Lamark T: Selective autophagy mediated by autophagic adapter proteins. Autophagy 7: 279-296, 2011.

32. Chen K, Yang YH, Jiang SD and Jiang LS: Decreased activity of osteocyte autophagy with aging may contribute to the bone loss in senile population. Histochem Cell Biol 142: 285-295, 2014.

33. Luo D, Ren H, Li T, Lian K and Lin D: Rapamycin reduces severity of senile osteoporosis by activating osteocyte autophagy. Osteoporos Int 27: 1093-1101, 2016.

34. Onal M, Piemontese M, Xiong J, Wang Y, Han L, Ye S, Komatsu M, Selig M, Weinstein RS, Zhao H, et al: Suppression of autophagy in osteocytes mimics skeletal aging. J Biol Chem 288: 17432-17440, 2013.

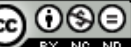

This work is licensed under a Creative Commons Attribution-NonCommercial-NoDerivatives 4.0 International (CC BY-NC-ND 4.0) License. 